\title{
Boundary Output Feedback Stabilization for a Cascaded-Wave PDE-ODE System with Velocity Recirculation
}

\author{
Ruicheng Li, Feng-Fei Jin (iD, and Baoqiang Yan \\ School of Mathematics and Statistics Shandong Normal University, Jinan 250014, China \\ Correspondence should be addressed to Feng-Fei Jin; jinfengfei@amss.ac.cn
}

Received 10 March 2021; Revised 4 May 2021; Accepted 25 May 2021; Published 4 June 2021

Academic Editor: Thach Ngoc Dinh

Copyright (c) 2021 Ruicheng Li et al. This is an open access article distributed under the Creative Commons Attribution License, which permits unrestricted use, distribution, and reproduction in any medium, provided the original work is properly cited.

\begin{abstract}
This paper considers the output feedback stabilization for a cascaded-wave PDE-ODE system with velocity recirculation by boundary control. First, we choose a well-known exponentially stable system as its target system and find a backstepping transformation to design a state feedback controller for the original system. Second, we attempt to give an output feedback controller for the original system by introducing the observer. The resulting closed-loop system admits a unique solution which is proved to be exponentially stable. Finally, we give some numerical examples to prove the validity for the theoretical results.
\end{abstract}

\section{Introduction}

In control engineering, ordinary differential equations (ODEs) and partial differential equations (PDEs) are widely used to model such problems. Many researchers have done detailed research on this content in the past decades. Systems modeled by ODEs are common in this respect. On the other hand, there are more and more works that contributed to stabilization for systems described by PDEs in recent years ([1-7]). For instance, aiming at solving the problem of system with variable coefficients and Neumann boundary actuation, $\mathrm{Wu}$ et al. used the backstepping approach that converted the wave equation into a same type equation in [8]. Not only that, the coupled PDE-ODE problem was also studied in control systems, where PDEs mainly included heat equations and wave equations. With the development of research on such problems, more results of PDE-ODE emerged. For example, in [9-16], the authors solved the stabilization for heat PDE-ODE by boundary control systems which used the PDE backstepping method. In $[10,13]$, Tang and Xie considered the systems where $\zeta(l, t)=U(t)$ in boundary of "PDE part." The system of $\zeta(l, t)=U(t-d)$ in "PDE part" was considered in [12]. The stabilization for wave PDE-ODE systems by the PDE backstepping method was solved in $[6,7,17,18]$. But beyond that, in [19],
Meglio solved the problem of stabilizing a linear ODE coupled first-order hyperbolic PDE, which was also based on the backstepping approach. This paper proposed a unified framework of iterative learning control for typical flexible structures under spatiotemporally varying disturbances in [20]. In [21], the control problem was addressed for a hybrid PDE-ODE system that described a nonuniform gantry crane system with constrained tension. In addition, the PDE-ODE models also played a significant role in practical application, such as 3D-printing [22], oil drilling [23], cable elevator [24], battery management [25], traffic [26], and so on.

In fact, the backstepping method played an important role in solving many PDEs and PDE-ODE problems, which were introduced by Krstic [27-30]. This approach was dedicated to finding an invertible Volterra integral transformation which mapped the considered plant with the boundary feedback law into a known stable target system. About some PDE systems, Jin and Guo also used the backstepping method to solve the PDEs system of nonlocal terms in [31]. In [32], Hasan and Tang used the backstepping method to model boundary stabilization of the Korteweg-De Vries (KdV) equation with sensors and actuators. For some PDE-ODE systems, the authors also used the backstepping approach to design the controller in $[6,7,9-18]$. In [17], Zhou et al. used directly the known 
backstepping method (from [30]) to achieve the stabilization of system matched disturbance with boundary control. It can be said that backstepping is a significant method and key move to deal with such PDE and PDEODE problems.

In this paper, our main focus is on the output feedback stabilization for a cascaded-wave PDE-ODE system subject to boundary control with velocity recirculation:

$$
\left\{\begin{array}{l}
\dot{X}(t)=D X(t)+F \zeta(0, t), \quad t>0, \\
\zeta_{t t}(x, t)=\zeta_{x x}(x, t)+q \zeta_{t}(0, t), \quad x \in(0,1), t>0, \\
\zeta_{x}(0, t)=0, \quad t \geq 0 \\
\zeta_{x}(1, t)=\bar{U}(t), \quad t \geq 0, \\
\zeta(x, 0)=\zeta_{0}(x) \\
\zeta_{t}(x, 0)=\zeta_{1}(x), \\
X(0)=X_{0}, \quad x \in[0,1], \\
y(t)=\left\{Q X(t), \zeta(0, t), \zeta_{t}(0, t)\right\}, \quad t \geq 0,
\end{array}\right.
$$

where $y(t)$ is the output (measurement), $\bar{U}(t) \in L_{\mathrm{loc}}^{2}(0, \infty)$ is the control input of the entire system, $\left(X_{0}, \zeta_{0}, \zeta_{1}\right)$ is the initial value, $q \in \mathbb{R}$ is a known constant, $D \in \mathbb{R}^{n \times n}$, and $F \in \mathbb{R}^{n \times 1}$. We suppose that $\sum(D, F)$ is stabilizable and $(Q, D)$ is observable. System $(1)$ is considered in the state space $\mathscr{H}=\mathbb{R}^{n} \times H^{1}(0,1) \times L^{2}(0,1)$.

On some special cases, we consider the following. For system (1) without " $X$ part," Su et al. gave the backstepping method and the state feedback controller in [33], which was $\zeta_{t t}(x, t)=\zeta_{x x}(x, t)+q \zeta_{t}(0, t)$. When $q=0$, by the backstepping method shown in [30], one can easily design a control law to stabilize system (1). And in [16], Zhou and Xu used backstepping transformation to settle the problem of coupled PDE-ODE where there was no non-local term in heat equation. On such coupling problems, there was few research on PDE-ODE problem with non-local term. Nonlocal terms, including both boundary terms and strict feedback/Volterra terms, have been the mainstay of the applications of PDE backstepping methods to parabolic PDEs. Equation $\zeta_{t t}(x, t)=\zeta_{x x}(x, t)+q u_{t}\left(x_{0}, t\right)$ models the string vibration of an electric guitar with a pickup at the location $x_{0} \in[0,1]$. The move of $x_{0}$ leads to the location change of the pickup. Inspired by it, we let $q=0$ in $\zeta_{t t}(x, t)=\zeta_{x x}(x, t)+q u_{t}\left(x_{0}, t\right)$. Compared with [30], we add a non-local term $q \zeta_{t}(0, t)$ to the right end of the wave equation. This makes it more difficult for us to research this problem. In [30], the form of backstepping transformation is “ $v(x, t)=u(x, t)-\int_{0}^{x} k(x, y) u(y, t) \mathrm{d} y-\int_{0}^{x} l(x, y) u_{t}(y, t)$ $\mathrm{d} y-\gamma(x) X(t)$ "; it does not work to solve the problem in their way. Inspired by (4) in [34], we obtain the new form of backstepping transformation. Using the backstepping and introducing the exponentially stable target system to design state feedback controller are also nice methods in this paper.

The paper is organized as follows. We design the state feedback controller for system (1) in Section 2. Section 3 is contributed to designing the output feedback control and proving the exponential stability of the closed-loop system.
In Section 4, we give some numerical examples to prove the validity of the proposed controller.

\section{State Feedback Controller Design}

In this section, we are devoted to designing a state feedback controller for system (1). First, we introduce the backstepping approach:

$$
\begin{aligned}
X(t)= & X(t), \\
v(x, t)= & \zeta(x, t)-\int_{0}^{x} k(x, y) \zeta(y, t) \mathrm{d} y \\
& -\int_{0}^{x} l(x, y) \zeta_{t}(y, t) \mathrm{d} y-\mu(x) \zeta(0, t)-\gamma(x) X(t),
\end{aligned}
$$

where the kernel functions $k(x, y), l(x, y), \mu(x)$, and $\gamma(x)$ will be defined later. Compared with the backstepping in [30], we add an additional term " $-\mu(x) \zeta(0, t)$ " in transformation (2). And we hope that turns system (1) into the target system

$$
\left\{\begin{array}{l}
\dot{X}(t)=(D+F G) X(t)+F v(0, t), \\
v_{t t}(x, t)=v_{x x}(x, t), \\
v_{x}(0, t)=0 \\
v_{x}(1, t)=-c_{1} v_{t}(1, t)-c_{2} v(1, t), \\
v(x, 0)=v_{0} \\
v_{t}(x, 0)=v_{1} \\
X(0)=X_{0}
\end{array}\right.
$$

where $c_{1}, c_{2}>0$ are tuning parameters and $G$ is chosen such that $D+F G$ is Hurwitz.

It is well known that there exists a exponentially stable solution for " $v$ part" in the state space $\mathscr{H}_{1}=H^{1}(0,1) \times L^{2}$ $(0,1)$, which is equipped with the norm induced by inner product

$$
\begin{aligned}
\left\langle\left(f_{1}, g_{1}\right)^{\top},\left(f_{2}, g_{2}\right)^{\top}\right\rangle_{\mathscr{H}_{1}}= & \int_{0}^{1}\left[f_{1}^{\prime}(x) f_{2}^{\prime}(x)+g_{1}(x) g_{2}(x)\right] \mathrm{d} x \\
& +c_{2} f_{1}(1) f_{2}(1), \quad \forall\left(f_{1}, g_{1}\right)^{\top}, \\
& \cdot\left(f_{2}, g_{2}\right)^{\top} \in \mathscr{H}_{1} .
\end{aligned}
$$

In order to get these kernel functions, we make the following calculation. Taking derivative of (2) with respect to $x$, we can obtain

$$
\begin{aligned}
v_{x}(x, t)= & \zeta_{x}(x, t)-k(x, x) \zeta(x, t) \\
& -\int_{0}^{x} k_{x}(x, y) \zeta(y, t) \mathrm{d} y-l(x, x) \zeta_{t}(x, t) \\
& -\int_{0}^{x} l_{x}(x, y) \zeta_{t}(y, t) \mathrm{d} y \\
& -\mu^{\prime}(x) \zeta(0, t)-\gamma^{\prime}(x) X(t) .
\end{aligned}
$$

Taking derivative of (2) with respect to $x$ twice, we find 


$$
\begin{aligned}
v_{x x}(x, t)= & \zeta_{x x}(x, t)-\frac{\mathrm{d}}{\mathrm{d} x} k(x, x) \zeta(x, t)-k(x, x) \zeta_{x}(x, t)-k_{x}(x, x) \zeta(x, t)-\int_{0}^{x} k_{x x}(x, y) \zeta(y, t) \mathrm{d} y \\
& -\frac{\mathrm{d}}{\mathrm{d} x} l(x, x) \zeta_{t}(x, t)-l(x, x) \zeta_{x t}(x, t)-l_{x}(x, x) \zeta_{t}(x, t)-\int_{0}^{x} l_{x x}(x, y) \zeta_{t}(y, t) \mathrm{d} y-\mu^{\prime \prime}(x) \zeta(0, t)-\gamma^{\prime \prime}(x) X(t) .
\end{aligned}
$$

In the same way, taking derivative of (2) with respect to $t$ and taking derivative of (2) with respect to $t$ twice and using the partial integral method, we can get the following:

$$
\begin{aligned}
\dot{X}(t)= & D X(t)+F \zeta(0, t), \\
v_{t}(x, t)= & \zeta_{t}(x, t)-\int_{0}^{x} k(x, y) \zeta_{t}(y, t) \mathrm{d} y-\int_{0}^{x} l(x, y) \zeta_{t t}(y, t) \mathrm{d} y-\mu(x) \zeta_{t}(0, t)-\gamma(x) \dot{X}(t) \\
= & \zeta_{t}(x, t)-\int_{0}^{x} k(x, y) \zeta_{t}(y, t) \mathrm{d} y-l(x, x) \zeta_{x}(x, t)+l_{y}(x, x) \zeta(x, t)-l_{y}(x, 0) \zeta(0, t)-\int_{0}^{x} l_{y y}(x, y) \zeta(y, t) \mathrm{d} y \\
& -q \zeta_{t}(0, t) \int_{0}^{x} l(x, y) \mathrm{d} y-\mu(x) \zeta_{t}(0, t)-\gamma(x) D X(t)-\gamma(x) F \zeta(0, t), \\
\ddot{X}(t)= & D^{2} X(t)+D F \zeta(0, t)+F \zeta_{t}(0, t), \\
v_{t t}(x, t)= & \zeta_{t t}(x, t)-\int_{0}^{x} k(x, y) \zeta_{t t}(y, t) \mathrm{d} y-\int_{0}^{x} l(x, y) \zeta_{t t t}(y, t) \mathrm{d} y-\mu(x) \zeta_{t t}(0, t)-\gamma(x) \ddot{X}(t) \\
= & \zeta_{x x}(x, t)+q \zeta_{t}(0, t)-k(x, x) \zeta_{x}(x, t)+k_{y}(x, x) \zeta(x, t)-k_{y}(x, 0) \zeta(0, t)-\int_{0}^{x} k_{y y}(x, y) \zeta(y, t) \mathrm{d} y \\
& -q \zeta_{t}(0, t) \int_{0}^{x} k(x, y) \mathrm{d} y-l(x, x) \zeta_{x t}(x, t)+l_{y}(x, x) \zeta_{t}(x, t)-l_{y}(x, 0) \zeta_{t}(0, t)-\int_{0}^{x} l_{y y}(x, y) \zeta_{t}(y, t) \mathrm{d} y \\
& -q \zeta_{t t}(0, t) \int_{0}^{x} l(x, y) \mathrm{d} y-\mu(x) \zeta_{t t}(0, t)-\gamma(x) D^{2} X(t)-\gamma(x) D F \zeta(0, t)-\gamma(x) F \zeta_{t}(0, t) .
\end{aligned}
$$

Then, we have

$$
\begin{aligned}
v_{t t}(x, t)-v_{x x}(x, t)= & {\left[\frac{\mathrm{d}}{\mathrm{d} x} l(x, x)+l_{x}(x, x)+l_{y}(x, x)\right] \zeta_{t}(x, t)+\left[\frac{\mathrm{d}}{\mathrm{d} x} k(x, x)+k_{x}(x, x)+k_{y}(x, x)\right] \zeta(x, t) } \\
& +\int_{0}^{x}\left[k_{x x}(x, y)-k_{y y}(x, y)\right] \zeta(y, t) \mathrm{d} y+\int_{0}^{x}\left[l_{x x}(x, y)-l_{y y}(x, y)\right] \zeta_{t}(y, t) \mathrm{d} y-\left[k_{y}(x, 0)\right. \\
& \left.+\gamma(x) D F-\mu^{\prime \prime}(x)\right] \zeta(0, t)+\left[\gamma^{\prime \prime}(x)-\gamma(x) D^{2}\right] X(t)-\left[q \int_{0}^{x} l(x, y) \mathrm{d} y+\mu(x)\right] \zeta_{t t}(0, t) \\
& +\left[q-q \int_{0}^{x} k(x, y) \mathrm{d} y-l_{y}(x, 0)-\gamma(x) F\right] \zeta_{t}(0, t)=0 .
\end{aligned}
$$
obtain

By the boundary condition $v_{x}(0, t)=0$ in (3) and (5), we

$$
\begin{aligned}
v_{x}(0, t) & =-k(0,0) \zeta(0, t)-l(0,0) \zeta_{t}(0, t)-\gamma^{\prime}(0) X(t)-\mu^{\prime}(0) \zeta(0, t) \\
& =\left[-k(0,0)-\mu^{\prime}(0)\right] \zeta(0, t)-l(0,0) \zeta_{t}(0, t)-\gamma^{\prime}(0) X(t) \\
& =0
\end{aligned}
$$


Hence, we choose the functions $k(x, y), l(x, y)$, $\gamma(x), \mu(x)$ to satisfy

$$
\left\{\begin{array}{l}
\gamma^{\prime \prime}(x)=\gamma(x) D^{2}, \\
\gamma^{\prime}(0)=0, \\
\gamma(0)=G, \\
k_{x x}(x, y)=k_{y y}(x, y), \\
k(x, x)=0, \\
k_{y}(x, 0)=-\gamma(x) D F-\mu^{\prime \prime}(x), \\
l_{x x}(x, y)=l_{y y}(x, y), \\
l(x, x)=0, \\
l_{y}(x, 0)=q-q \int_{0}^{x} k(x, y) \mathrm{d} y-\gamma(x) F, \\
q \int_{0}^{x} l(x, y) \mathrm{d} y+\mu(x)=0 .
\end{array}\right.
$$

We can obtain $\mu(0)=0$ by $q \int_{0}^{x} l(x, y) \mathrm{d} y+\mu(x)=0$. In [30], we find that the solution of $\gamma(x)$ is as follows:

$$
\gamma(x)=G\left(\begin{array}{ll}
I & 0
\end{array}\right) e^{\left(\begin{array}{cc}
0 & D^{2} \\
I & 0
\end{array}\right) x}\left(\begin{array}{l}
I \\
0
\end{array}\right) .
$$

By (2) and (10), we have $\zeta(0, t)=v(0, t)-\mu \zeta(0$, t) $-\gamma(0) X(t)=v(0, t)+G X(t)$; then, the first equation in (3) is obtained.

Next we can let $k(x, y)=k_{1}(x-y), l(x, y)=l_{1}(x-y)$; by " $k$ part" in (10), we can have

$$
\begin{aligned}
k_{y}(x, 0) & =-k_{1}^{\prime}(x)=-\gamma(x) D F-\mu^{\prime \prime}(x), \\
k_{1}(x) & =\int_{0}^{x} \gamma(\xi) \mathrm{d} \xi D F+\mu^{\prime \prime}(x) \\
& =\int_{0}^{x} \gamma(\xi) \mathrm{d} \xi D F+\mu^{\prime}(x)-\mu^{\prime}(0) .
\end{aligned}
$$

Through $q \int_{0}^{x} l(x, y) \mathrm{d} y+\mu(x)=0$, we get

$$
\begin{aligned}
\mu(x) & =-q \int_{0}^{x} l(x, y) \mathrm{d} y, \\
\mu^{\prime}(x) & =-q \int_{0}^{x} l_{x}(x, y) \mathrm{d} y=-q \int_{0}^{x} l_{1}^{\prime}(x-y) \mathrm{d} y \\
& =q l_{1}(0)-q l_{1}(x) .
\end{aligned}
$$

Because $\mu^{\prime}(x)=-q l_{1}(x), l(x, x)=0$, we can acquire $l(0,0)=l_{1}(0)=0, \mu^{\prime}(0)=0$. According to the above formulas, we obtain

$$
\begin{aligned}
k_{1}(x)= & \int_{0}^{x} \gamma(\xi) \mathrm{d} \xi D F-q \int_{0}^{x} \gamma(\xi) \mathrm{d} \xi F+q^{2} x \\
& -q^{2} \int_{0}^{x} \int_{0}^{z} k_{1}(z-y) \mathrm{d} y \mathrm{~d} z, \\
l_{1}(x)= & -q x+q \int_{0}^{x} \int_{0}^{z} k_{1}(z-y) \mathrm{d} y \mathrm{~d} z+\int_{0}^{x} \gamma(\xi) \mathrm{d} \xi F,
\end{aligned}
$$

$$
\mu(x)=-q \int_{0}^{x} l(x, y) \mathrm{d} y=-q \int_{0}^{x} l_{1}(x-y) \mathrm{d} y .
$$

According to $k(x, y)=k_{1}(x-y), l(x, y)=l_{1}(x-y)$, simplify the above three formulas in (14)(16). Let $\delta=z-$ $y, \vartheta=x-y$; we get $\int_{0}^{x} \int_{0}^{z} k_{1}(z-y) \mathrm{d} y \mathrm{~d} z=\int_{0}^{x} \int_{0}^{z} k_{1}(\delta) \mathrm{d} \delta$ $\mathrm{d} z, \int_{0}^{x} l_{1}(x-y) \mathrm{d} y=\int_{0}^{x} l_{1}(9) \mathrm{d} \vartheta$, and

$$
\begin{aligned}
k_{1}(x)= & \int_{0}^{x} \gamma(\xi) \mathrm{d} \xi D F-q \int_{0}^{x} \gamma(\xi) \mathrm{d} \xi F+q^{2} x \\
& -q^{2} \int_{0}^{x} \int_{0}^{z} k_{1}(\delta) \mathrm{d} \delta \mathrm{d} z, \\
l_{1}(x)= & -q x+q \int_{0}^{x} \int_{0}^{z} k_{1}(\delta) \mathrm{d} \delta \mathrm{d} z+\int_{0}^{x} \gamma(\xi) \mathrm{d} \xi F \\
= & -q x+q \int_{0}^{x}(x-\delta) k_{1}(\delta) \mathrm{d} \delta+\int_{0}^{x} \gamma(\xi) \mathrm{d} \xi F, \\
\mu(x)= & -q \int_{0}^{x} l_{1}(\vartheta) \mathrm{d} \vartheta .
\end{aligned}
$$

By differentiating (17) with respect to $x$ twice, we transform (17) into a second-order linear ODE and get the solution of $k_{1}(x)$ :

$$
\begin{aligned}
k_{1}(x)= & {\left[\frac{k}{q}(D F-q F)+q\right] \sin q x-\frac{\cos q x}{q} \int_{0}^{x} \sin q \xi } \\
& \cdot \gamma^{\prime}(\xi) \mathrm{d} \xi(D F-q F) \\
& +\frac{\sin q x}{q} \int_{0}^{x} \cos q \xi \cdot \gamma^{\prime}(\xi) \mathrm{d} \xi(D F-q F) .
\end{aligned}
$$

We substitute (20) for (18) and obtain the solution of $l_{1}(x)$. In the same way, the solution of $\mu(x)$ can also be obtained.

Because $\quad \gamma(\cdot) \in C^{1}[0,1], \quad \int_{0}^{x} \gamma(\xi) \mathrm{d} \xi D F \in C^{2}[0,1]$. $\int_{0}^{x} \gamma(\xi) \mathrm{d} \xi F \in C^{2}[0,1], \quad q^{2} x \in C^{2}[0,1], \quad k_{1}(\delta) \in C[0,1]$, so $k_{1}(\cdot) \in C^{2}[0,1]$, and $l_{1}(\cdot), \mu(\cdot) \in C^{2}[0,1]$.

We suppose that the inverse transformation of (2) exists in the form

$$
\begin{aligned}
X(t)= & X(t), \\
\zeta(x, t)= & v(x, t)+\int_{0}^{x} m(x, y) v(y, t) \mathrm{d} y \\
& +\int_{0}^{x} n(x, y) v_{t}(y, t) \mathrm{d} y+h(x) X(t),
\end{aligned}
$$

where $m(x, y), n(x, y), h(x)$ are the kernel functions.

Derive (21) with respect to $x$, and we obtain

$$
\begin{aligned}
\zeta_{x}(x, t)= & v_{x}(x, t)+m(x, x) v(x, t) \\
& +\int_{0}^{x} m_{x}(x, y) v(y, t) \mathrm{d} y+n(x, x) v_{t}(x, t) \\
& +\int_{0}^{x} n_{x}(x, y) v_{t}(y, t) \mathrm{d} y+h^{\prime}(x) X(t) .
\end{aligned}
$$

Differentiating (21) with respect to $x$ twice, we have 


$$
\begin{aligned}
\zeta_{x x}(x, t)= & v_{x x}(x, t)+\frac{\mathrm{d}}{\mathrm{d} x} m(x, x) v(x, t)+m(x, x) v_{x}(x, t) \\
& +m_{x}(x, x) v(x, t)+\int_{0}^{x} m_{x x}(x, y) v(y, t) \mathrm{d} y \\
& +\frac{\mathrm{d}}{\mathrm{d} x} n(x, x) v_{t}(x, t)+n(x, x) v_{x t}(x, t)+n_{x}(x, x) v_{t}(x, t) \\
& +\int_{0}^{x} n_{x x}(x, y) v_{t}(y, t) \mathrm{d} y-h^{\prime \prime}(x) X(t) .
\end{aligned}
$$

We differentiate (21) with respect to $t$ and get

$$
\begin{aligned}
\dot{X}(t)= & (D+F G) X(t)+F v(0, t), \\
\zeta_{t}(x, t)= & v_{t}(x, t)+\int_{0}^{x} m_{x}(x, y) v_{t}(y, t) \mathrm{d} y+\int_{0}^{x} n(x, y) v_{t t}(y, t) \mathrm{d} y+h(x) \dot{X}(t) \\
= & v_{t}(x, t)+\int_{0}^{x} m_{x}(x, y) v_{t}(y, t) \mathrm{d} y+n(x, x) v_{x}(x, t)-n_{y}(x, x) v(x, t)+n_{y}(x, 0) v(0, t) \\
& +\int_{0}^{x} n_{y y}(x, y) v(y, t) \mathrm{d} y+h(x)(D+F G) X(t)+h(x) F v(0, t) .
\end{aligned}
$$

Differentiating (21) twice on both sides with respect to $t$ yields

$$
\begin{aligned}
\ddot{X}(t) & =(D+F G)^{2} X(t)+(D+F G) F v(0, t)+F v_{t}(0, t), \\
\zeta_{t t}(x, t) & =v_{t t}(x, t)+\int_{0}^{x} m(x, y) v_{t t}(y, t) \mathrm{d} y+\int_{0}^{x} n(x, y) v_{t t t}(y, t) \mathrm{d} y+h(x) \ddot{X}
\end{aligned}
$$

Then, we have

$$
\begin{aligned}
\zeta_{t t}(x, t)-\zeta_{x x}(x, t)= & \int_{0}^{x}\left[m_{y y}(x, y)-m_{x x}(x, y)\right] v(y, t) \mathrm{d} y+\int_{0}^{x}\left[n_{y y}(x, y)-n_{x x}(x, y)\right] v_{t}(y, t) \mathrm{d} y \\
& -\left[\frac{\mathrm{d}}{\mathrm{d} x} m(x, x)+m_{x}(x, x)+m_{y}(x, x)\right] v(x, t) \\
& -\left[\frac{\mathrm{d}}{\mathrm{d} x} n(x, x)+n_{x}(x, x)+n_{y}(x, x)\right] v_{t}(x, t) \\
& +\left[m_{y}(x, 0)+h(x)(D+F G) F\right] v(0, t)+\left[n_{y}(x, 0)+h(x) F\right] v_{t}(0, t) \\
& +\left[h(x)(D+F G)^{2}-h^{\prime \prime}(x)\right] X(t) \\
= & 0 .
\end{aligned}
$$


The boundary condition at $x=0$ in (1) implies $\zeta_{x}(0, t)=m(0,0) v(0, t)+n(0,0) v_{t}(0, t)+h^{\prime}(0) X(t)=0$.

Hence, we choose the functions $m(x, y), n(x, y), l(x)$ to satisfy

$$
\left\{\begin{array}{l}
h^{\prime \prime}(x)=h(x)(D+F G)^{2}, \\
h^{\prime}(0)=0 \\
h(0)=G \\
m_{x x}(x, y)=m_{y y}(x, y), \\
m(x, x)=0 \\
m_{y}(x, 0)=-h(x)(D+F G) F \\
n_{x x}(x, y)=n_{y y}(x, y) \\
n(x, x)=0 \\
n_{y}(x, 0)=-h(x) F .
\end{array}\right.
$$

We obtain the solution of $h(x)$ in [30]:

$$
h(x)=G\left(\begin{array}{ll}
I & 0
\end{array}\right) e^{\left(\begin{array}{cc}
0 & (D+F G)^{2} \\
I & 0
\end{array}\right) x}\left(\begin{array}{l}
I \\
0
\end{array}\right) .
$$

Let $m(x, y)=m_{1}(x-y), n(x, y)=n_{1}(x-y)$; then,

$$
\begin{aligned}
m_{y}(x, 0) & =-m_{1}^{\prime}(x)=-h(x)(D+F G) F, \\
m_{1}(x) & =\int_{0}^{x} h(\xi) \mathrm{d} \xi(D+F G) F, \\
n_{y}(x, 0) & =-n_{1}^{\prime}(x)=-h(x) F, \\
n_{1}(x) & =\int_{0}^{x} h(\xi) \mathrm{d} \xi F .
\end{aligned}
$$

Combining the forth equations in (1) and (3), we can obtain the controller $\bar{U}$ for (1)

$$
\begin{aligned}
\bar{U}(t)= & \int_{0}^{1} k_{x}(1, y) \zeta(y, t) \mathrm{d} y+\int_{0}^{1} l_{x}(1, y) \zeta_{t}(y, t) \mathrm{d} y+\mu^{\prime}(1) \zeta(0, t)+\gamma^{\prime}(1) X(t)-c_{1}\left[\zeta_{t}(1, t)-\int_{0}^{1} k(1, y) \zeta_{t}(y, t) \mathrm{d} y\right. \\
& +l_{y}(1,1) \zeta(1, t)-l_{y}(1,0) \zeta(0, t)-\int_{0}^{1} l_{y y}(1, y) \zeta(y, t) \mathrm{d} y-q \zeta_{t}(0, t) \int_{0}^{1} l(1, y) \mathrm{d} y-\mu(1) \zeta_{t}(0, t)-\gamma(1) D X(t) \\
& -\gamma(1) F \zeta(0, t)]-c_{2}\left[\zeta(1, t)-\int_{0}^{1} k(1, y) \zeta(y, t) \mathrm{d} y-\int_{0}^{1} l(1, y) \zeta_{t}(y, t) \mathrm{d} y-\mu(1) \zeta(0, t)-\gamma(1) X(t)\right] \\
= & \int_{0}^{1}\left[k_{x}(1, y)+c_{1} l_{y y}(1, y)+c_{2} k(1, y)\right] \zeta(y, t) \mathrm{d} y+\int_{0}^{1}\left[l_{x}(1, y)+c_{1} k(1, y)+c_{2} l(1, y)\right] \zeta_{t}(y, t) \mathrm{d} y \\
& +\left[\mu^{\prime}(1)+c_{1} l_{y}(1,0)+c_{1} \gamma(1) F+c_{2} \mu(1)\right] \zeta(0, t)+\left[c_{1} q \int_{0}^{1} l(1, y) d y+c_{1} \mu(1)\right] \zeta_{t}(0, t) \\
& -\left[c_{1} l_{y}(1,1)+c_{2}\right] \zeta(1, t)-c_{1} \zeta_{t}(1, t)+\left[c_{1} \gamma(1) D+c_{2} \gamma(1)+\gamma^{\prime}(1)\right] X(t) .
\end{aligned}
$$

For convenience, we describe system (1) under controller (31) as

$$
\left\{\begin{array}{l}
\dot{X}(t)=D X(t)+F \zeta(0, t) \\
\zeta_{t t}(x, t)=\zeta_{x x}(x, t)+q \zeta_{t}(0, t) \\
\zeta_{x}(0, t)=0 \\
\zeta_{x}(1, t)=\int_{0}^{1}\left[k_{x}(1, y)+c_{1} l_{y y}(1, y)+c_{2} k(1, y)\right] \zeta(y, t) \mathrm{d} y+\int_{0}^{1}\left[l_{x}(1, y)+c_{1} k(1, y)+c_{2} l(1, y)\right] \zeta_{t}(y, t) \mathrm{d} y \\
+\left[\mu^{\prime}(1)+c_{1} l_{y}(1,0)+c_{1} \gamma(1) F+c_{2} \mu(1)\right] \zeta(0, t)+\left[c_{1} q \int_{0}^{1} l(1, y) \mathrm{d} y+c_{1} \mu(1)\right] \zeta_{t}(0, t) \\
-\left[c_{1} l_{y}(1,1)+c_{2}\right] \zeta(1, t)-c_{1} \zeta_{t}(1, t)+\left[c_{1} \gamma(1) D+c_{2} \gamma(1)+\gamma^{\prime}(1)\right] X(t) .
\end{array}\right.
$$


Define the state space $\mathscr{H}=\mathbb{R}^{n} \times H^{1}(0,1) \times L^{2}(0,1)$ for system (32) with the norm

$$
\begin{aligned}
\|(X, f, g)\|_{\mathscr{C}}^{2}= & \int_{0}^{1}\left(\left|f^{\prime}(x)\right|^{2}+|g(x)|^{2}\right) \mathrm{d} x \\
& +k_{1}\|X\|_{\mathbb{R}^{n}}^{2}+k_{2}|f(0)|^{2}, \quad \forall(X, f, g) \in \mathscr{H},
\end{aligned}
$$

where $k_{1}, k_{2}>0$.

Theorem 1. Assume that $c_{1}, c_{2}>0$ and $G$ is chosen such that $D+F G$ is Hurwitz. For any initial condition such that $\left(X(0), \zeta(\cdot, 0), \zeta_{t}(\cdot, 0)\right)^{\top} \in \mathscr{H}$, closed-loop system (32) admits a unique solution $\left(X(t), \zeta(\cdot, t), \quad \zeta_{t}(\cdot, t)\right)^{\top} \in C \quad(0, \infty ; \mathscr{H})$. Moreover, closed-loop system (32) is exponentially stable: there exist two positive constants $M, \varepsilon$ depending on $c_{1}, c_{2}$ and G such that

$$
\left\|\left(X(t), \zeta(\cdot, t), \zeta_{t}(\cdot, t)\right)^{\top}\right\|_{\mathscr{H}} \leq M e^{-\varepsilon t}\left\|\left(X_{0}, \zeta_{0}, \zeta_{1}\right)^{\top}\right\|_{\mathscr{H}}, \quad \forall t \geq 0 .
$$

Proof. It is a direct result from the equivalence between system (3) and (32). Obviously, we define the operator $\mathscr{A}_{1}: D\left(\mathscr{A}_{1}\right) \longrightarrow \mathscr{H}_{1}$ for the " $v$ part" of target system (3):

$$
\left\{\begin{array}{l}
\mathscr{A}_{1}(f, g)^{\top}=\left(g, f^{\prime \prime}\right)^{\top}, \\
D\left(\mathscr{A}_{1}\right)=\left\{(f, g)^{\top} \in H^{2}(0,1) \times H^{1}(0,1) \mid \mathscr{A}_{1}(f, g)^{\top} \in \mathscr{H}_{1}, f^{\prime}(0)=0, f^{\prime}(1)=-c_{1} g(1)-c_{2} f(1)\right\} .
\end{array}\right.
$$

Then the " $v$ part" of system (3) can be written as an abstract evolutionary equation in $\mathscr{H}_{1}=H^{1}(0,1) \times L^{2}(0,1)$ :

$$
\frac{\mathrm{d}}{\mathrm{d} t}\left(v(\cdot, t), v_{t}(\cdot, t)\right)^{\top}=\mathscr{A}_{1}\left(v(\cdot, t), v_{t}(\cdot, t)\right)^{\top} .
$$

As we all know, the operator $\mathscr{A}_{1}$ can generate an exponentially stable $C_{0}$-semigroup $e^{\mathscr{A}_{1} t}$ on $\mathscr{H}_{1}$ [35]. In other words, there exist $M_{1}, \alpha>0$ such that

$$
\left\|e^{\mathscr{A} t_{1} t}\right\| \leq M_{1} e^{-\alpha t}, \quad \forall t>0 .
$$

Next we consider the " $X$ part" of target system (3) on $\mathbb{R}^{n}$. Because $v(0, t) \in C(0, \infty)$, using the constant variational formula, the solution of " $X$ part" is given by

$$
X(t)=e^{(D+F G) t} X(0)+\int_{0}^{t} e^{(D+F G)(t-s)} F v(0, s) \mathrm{d} s .
$$

Because $D+F G$ is Hurwitz, there exist positive constants $C_{5}, \theta>0$ such that

$$
\left\|e^{(D+F G) t}\right\| \leq C_{5} e^{-\theta t} .
$$

From Theorem 1 in [17], the solution of " $X$ part" is exponentially stable. So, system (3) admits a unique solution and the solution is exponentially stable. According to transformations (2) and (21), we define a bounded invertible operator $P:(X, f, g) \longrightarrow(X, \varphi, \psi)$ in $\mathscr{H}:$

$$
\begin{aligned}
& \left(\begin{array}{c}
X \\
\varphi(x) \\
\psi(x)
\end{array}\right)=\left(\begin{array}{c}
X \\
f(x)-\int_{0}^{x} k(x, y) f(y) \mathrm{d} y-\int_{0}^{x} l(x, y) g(y) \mathrm{d} y-\mu(x) f(0)-\gamma(x) X \\
g(x)-\int_{0}^{x} k(x, y) g(y) \mathrm{d} y-l(x, x) f^{\prime}(x)+l_{y}(x, x) f(x)+t_{1}(x)
\end{array}\right) \\
& \left(\begin{array}{c}
X \\
f(x) \\
g(x)
\end{array}\right)=\left(\begin{array}{c}
X \\
\varphi(x)+\int_{0}^{x} m(x, y) \varphi(y) \mathrm{d} y+\int_{0}^{x} n(x, y) \psi(y) \mathrm{d} y+h(x) X \\
\psi(x)+\int_{0}^{x} m_{x}(x, y) \psi(y) \mathrm{d} y+n(x, x) \varphi^{\prime}(x)-n_{y}(x, x) \varphi(x)+t_{2}(x)
\end{array}\right),
\end{aligned}
$$

where

$$
\begin{aligned}
& t_{1}(x)=-l_{y}(x, 0) f(0)-\int_{0}^{x} l_{y y}(x, y) f(y) \mathrm{d} y-q g(0) \int_{0}^{x} l(x, y) \mathrm{d} y-\mu(x) g(0)-\gamma(x) D X+\gamma(x) F f(0), \\
& t_{2}(x)=n_{y}(x, 0) \varphi(0)+\int_{0}^{x} n_{y y}(x, y) \varphi(y) \mathrm{d} y+h(x)(D+F G) X+h(x) F \varphi(0),
\end{aligned}
$$


and $k(x, y), l(x, y), \mu(x), \gamma(x), m(x, y), n(x, y), h(x)$ are defined in (14)(16) and (30), respectively.

By the formula above, we have the following expression:

$$
\begin{aligned}
\left\|(X, f(x), g(x))^{\top}\right\| & \leq\left\|P^{-1}\right\|\left\|(X, \varphi(x), \psi(x))^{\top}\right\| \\
& \leq M_{2}\left\|(X, \varphi(x), \psi(x))^{\top}\right\| .
\end{aligned}
$$

Since the operator $P$ is bounded and invertible, there exists $M_{2}>0$ such that $\left\|P^{-1}\right\|<M_{2}$. Besides, the " $v$ part" admits a unique solution and the solution is exponentially stable, so the solution of closed-loop system (32) is exponentially stable by (42). There exist $M, \varepsilon>0$ such that (34) holds.

\section{Observer and Output Feedback Controller Design}

In this section, we focus on designing output feedback controller for system (1) on the grounds of the observer. Next, we give the observer for system (1).

$$
\left\{\begin{array}{l}
\dot{\hat{X}}(t)=D \widehat{X}(t)+P(Q \widehat{X}-Q X)+F \zeta(0, t), \\
\widehat{\zeta}_{t t}(x, t)=\widehat{\zeta}_{x x}(x, t)+q \zeta_{t}(0, t), \\
\widehat{\zeta}_{x}(0, t)=c_{3}\left[\widehat{\zeta}_{t}(0, t)-\zeta_{t}(0, t)\right]+c_{4}[\widehat{\zeta}(0, t)-\zeta(0, t)] \\
\widehat{\zeta}_{x}(1, t)=\bar{U}(t), \\
\widehat{\zeta}_{(x, 0)=\widehat{\zeta}_{0},} \\
\widehat{\zeta}_{t}(x, 0)=\widehat{\zeta}_{1}, \\
\widehat{X}(0)=\widehat{X}_{0},
\end{array}\right.
$$

where $c_{3}, c_{4}$ are positive design parameters.

The error $\left(\widetilde{X}(t), \widetilde{\zeta}(x, t), \zeta_{t}(x, t)\right)=t(\widehat{X}(t)-X(t), \widehat{\zeta}(x$, $\left.t)-\zeta(x, t), \widehat{\zeta}_{t}(x, t)-\zeta_{t}(x, t)\right)$ between system (1) and observer (43) is governed by

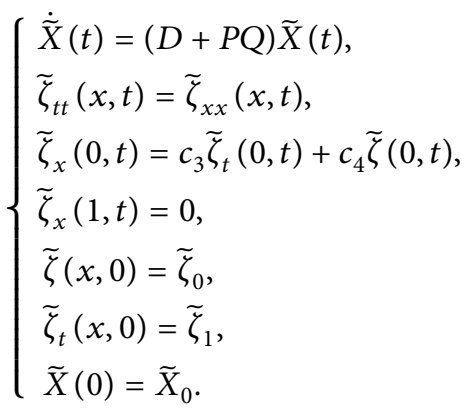

Define the state space $\mathscr{H}=\mathbb{R}^{n} \times H^{1}(0,1) \times L^{2}(0,1)$ for system (44) with the norm

$$
\begin{aligned}
\left\|\left(\tilde{X}, \widetilde{\zeta}, \widetilde{\zeta}_{t}\right)\right\|_{\mathscr{H}}^{2}= & \int_{0}^{1}\left(\left|\widetilde{\zeta}_{t}\right|^{2}+\left|\widetilde{\zeta}_{x}\right|^{2}\right) \mathrm{d} x+\|\widetilde{X}\|_{\mathbb{R}^{n}}^{2} \\
& +c_{4}|f(0)|^{2}, \quad \forall\left(\widetilde{X}, \widetilde{\zeta}, \widetilde{\zeta}_{t}\right) \in \mathscr{H} .
\end{aligned}
$$

Theorem 2. Suppose thatc ${ }_{3}, c_{4}>0$. For any initial value $\left(\widetilde{X}_{0}, \widetilde{\zeta}_{0}, \widetilde{\zeta}_{1}\right)^{\top} \in \mathscr{H}$, system 4 4admits a unique exponentially stable solution $\left(\widetilde{X}(t), \widetilde{\zeta}(\cdot, t), \widetilde{\zeta}_{t}(\cdot, t)\right)^{\top} \in C(0, \infty ; \mathscr{H})$ in the sense that

$$
E(t) \leq C_{1} E(0) e^{-\omega_{1} t},
$$

for some positive constants $C_{1}$ and $\omega_{1}$, whereE $(t)$ is given by

$$
E(t):=\frac{1}{2} \int_{0}^{1}\left(\widetilde{\zeta}_{x}^{2}(x, t)+\widetilde{\zeta}_{t}^{2}(x, t)\right) \mathrm{d} x+\frac{c_{4}}{2} \widetilde{\zeta}^{2}(1, t)+|\tilde{X}|_{\mathbb{R}^{n}}^{2}
$$

Moreover,

$$
|j(t)| \leq M_{0} e^{-\omega_{1} t},
$$

$$
\int_{t_{1}}^{t_{2}}|r(t)|^{2} \leq M_{1}\left(e^{-\omega_{1} t_{1}}+e^{-\omega_{1} t_{2}}\right), \quad \forall t_{2}>t_{1}>0,
$$

for $M_{0}, M_{1}>0$ and $f(\cdot) \in L^{2}(0, \infty)$, where

$$
\begin{aligned}
f(t)= & \int_{0}^{1}\left[k_{x}(1, y)+c_{1} l_{y y}(1, y)+c_{2} k(1, y)\right] \widetilde{\zeta}(y, t) \mathrm{d} y \\
& +\int_{0}^{1}\left[l_{x}(1, y)+c_{1} k(1, y)+c_{2} l(1, y)\right] \widetilde{\zeta}_{t}(y, t) \mathrm{d} y \\
& +\left[\mu^{\prime}(1)+c_{1} l_{y}(1,0)+c_{1} \gamma(1) F+c_{2} \mu(1)\right] \widetilde{\zeta}(0, t) \\
& +\left[c_{1} q \int_{0}^{1} l(1, y) d y+c_{1} \mu(1)\right] \widetilde{\zeta}_{t}(0, t) \\
& -\left[c_{1} l_{y}(1,1)+c_{2}\right] \widetilde{\zeta}(1, t)-c_{1} \widetilde{\zeta}_{t}(1, t)+\left[c_{1} \gamma(1) D+c_{2} \gamma(1)+\gamma^{\prime}(1)\right] \tilde{X}(t), \\
r(t)= & {\left[c_{1} q \int_{0}^{1} l(1, y) d y+c_{1} \mu(1)\right] \widetilde{\zeta}_{t}(0, t)-c_{1} \widetilde{\zeta}_{t}(1, t), } \\
j(t)= & f(t)-r(t) .
\end{aligned}
$$


Proof. As we all know, the " $\widetilde{\zeta}$ part" of system (44) admits a unique exponentially stable solution from [33] and $D+P Q$ is Hurwitz, so system (44) has a unique exponentially stable solution.

Notice that the energy $\left(E(t)-|\widetilde{X}|_{\mathbb{R}^{n}}^{2}\right)$ is equivalent to $\left\|\left(\widetilde{\zeta}(\cdot, t), \widetilde{\zeta}_{t}(\cdot, t)\right)^{\top}\right\|_{\mathscr{H}_{1}}^{2}$. We only prove $(48)$ and $(49)$. By Cauchy inequality and Hölder inequality, we have

$$
|j(t)|^{2} \leq C\left[\int_{0}^{1} \widetilde{\zeta}_{x}^{2}(y, t) \mathrm{d} y+\int_{0}^{1} \widetilde{\zeta}_{t}^{2}(y, t) \mathrm{d} y+\widetilde{\zeta}^{2}(0, t)+\widetilde{X}^{2}(t)\right],
$$

for some constant $C>0$.

$\sim_{2}$ It is obvious that $\int_{0}^{1} \widetilde{\zeta}_{x}^{2}(y, t) \mathrm{d} y+\int_{0}^{1} \widetilde{\zeta}_{t}^{2}(y, t) \mathrm{d} y+$ $\widetilde{\zeta}^{2}(0, t)+\widetilde{X}^{2}(t)$ is equivalent to $E(t)$. $E(t)$ is exponentially stable, so (48) holds. Next we prove (49).

Differentiating $E(t)$, we find

$$
\dot{E}(t)=-c_{3}\left|\widetilde{\zeta}_{t}(0, t)\right|^{2} \leq 0
$$

Integrating above equation from $t_{1}$ to $t_{2}$, we obtain

$$
\begin{aligned}
\int_{t_{1}}^{t_{2}}\left|\widetilde{\zeta}_{t}(0, t)\right|^{2} \mathrm{~d} x & =-\frac{1}{c_{3}} \int_{t_{1}}^{t_{2}} \dot{E}(s) \mathrm{d} s=\frac{1}{c_{3}}\left(E\left(t_{1}\right)-E\left(t_{2}\right)\right) \\
& \leq \frac{1}{c_{3}} C_{1} E(0)\left(e^{-\omega_{1} t_{1}}+e^{-\omega_{1} t_{2}}\right) .
\end{aligned}
$$

Define another function

$$
\rho(t)=\int_{0}^{1} x \widetilde{\zeta}_{t}(x, t) \widetilde{\zeta}_{x}(x, t) \mathrm{d} x
$$

It is clear that $|\rho(t)| \leq E(t)$. Taking derivative of (54), we get

$$
\dot{\rho}(t)=\frac{1}{2} \widetilde{\zeta}_{t}^{2}(1, t)-\frac{1}{2} \int_{0}^{1}\left(\widetilde{\zeta}_{t}^{2}(x, t)+\widetilde{\zeta}_{x}^{2}(x, t)\right) \mathrm{d} x .
$$

Integrating above equation from $t_{1}$ to $t_{2}$, we have

$$
\begin{aligned}
\frac{1}{2} \int_{t_{1}}^{t_{2}} \widetilde{\zeta}_{t}(1, t) \mathrm{d} t= & \rho\left(t_{2}\right)-\rho\left(t_{1}\right) \\
& +\frac{1}{2} \int_{t_{1}}^{t_{2}} \int_{0}^{1}\left(\widetilde{\zeta}_{t}^{2}(x, t)+\widetilde{\zeta}_{x}^{2}(x, t)\right) \mathrm{d} x \mathrm{~d} t \\
\leq & E\left(t_{2}\right)+E\left(t_{1}\right)+\int_{t_{1}}^{t_{2}} E(t) \mathrm{d} t \\
\leq & C_{2}\left(e^{-\omega_{1} t_{1}}+e^{-\omega_{1} t_{2}}\right),
\end{aligned}
$$

for some constant $C_{2}>0$. Hence, (48) and (49) hold. Especially, when $t_{1}=0, f(t) \in L^{2}(0, \infty)$.

We give the output feedback controller about system (1):

$$
\begin{aligned}
\bar{U}(t)= & \int_{0}^{1}\left[k_{x}(1, y)+c_{1} l_{y y}(1, y)+c_{2} k(1, y)\right] \widehat{\zeta}(y, t) \mathrm{d} y \\
& +\int_{0}^{1}\left[l_{x}(1, y)+c_{1} k(1, y)+c_{2} l(1, y)\right] \widehat{\zeta}_{t}(y, t) \mathrm{d} y \\
& +\left[\mu^{\prime}(1)+c_{1} l_{y}(1,0)+c_{1} \gamma(1) F+c_{2} \mu(1)\right] \widehat{\zeta}(0, t) \\
& +\left[c_{1} q \int_{0}^{1} l(1, y) \mathrm{d} y+c_{1} \mu(1)\right] \widehat{\zeta}_{t}(0, t) \\
& -\left[c_{1} l_{y}(1,1)+c_{2}\right] \widehat{\zeta}(1, t)-c_{1} \widehat{\zeta}_{t}(1, t) \\
& +\left[c_{1} \gamma(1) D+c_{2} \gamma(1)+\gamma^{\prime}(1)\right] \widehat{X}(t) .
\end{aligned}
$$

In terms of control law (57), closed-loop system (1) can be described as 


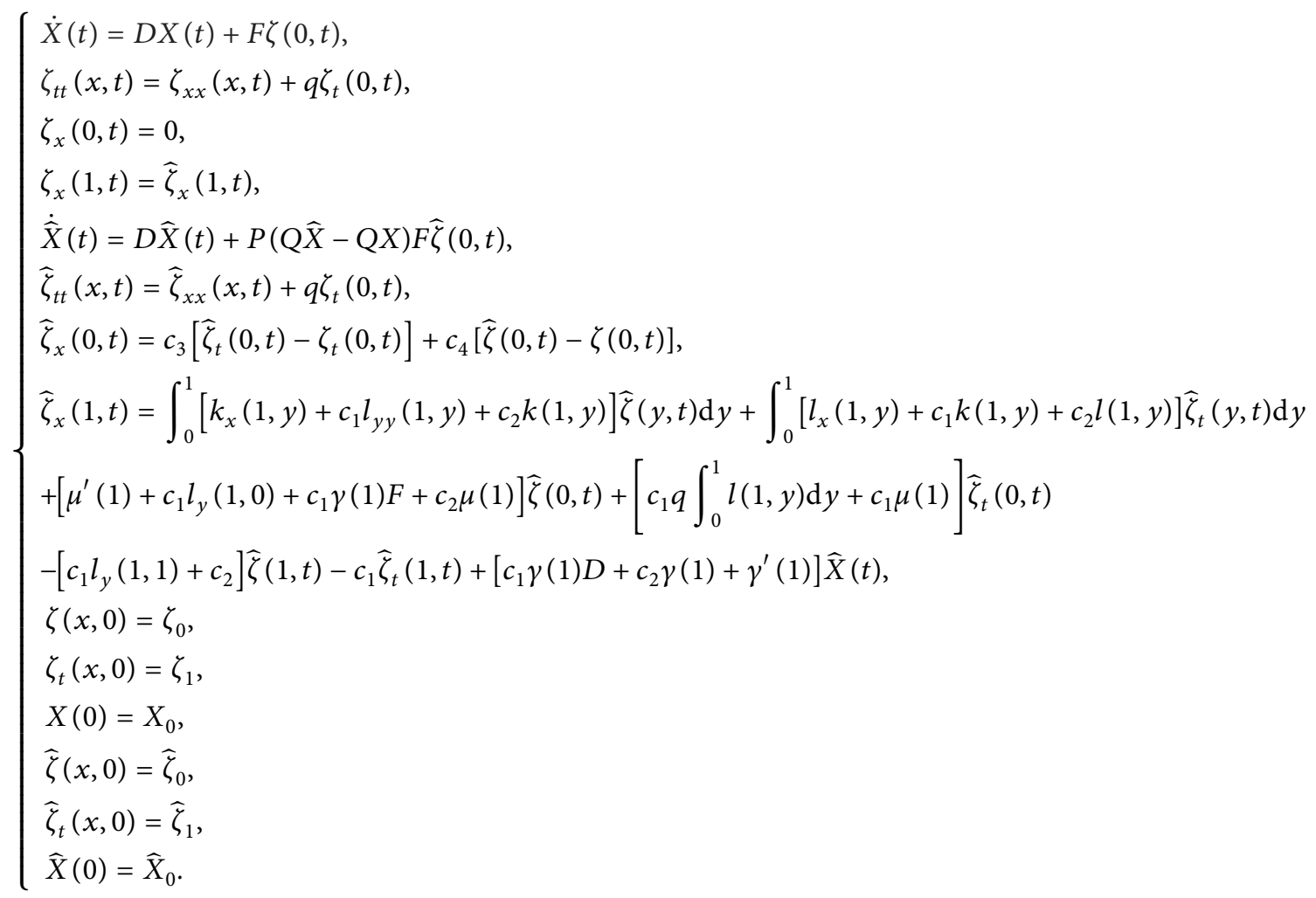

We consider system (58) in the state space $\mathscr{H}^{2}$.

Theorem 3. Assume thatc $c_{1}, c_{2}>0$. For any initial value $\left(X(0), \zeta(\cdot, 0), \zeta_{t}(\cdot, 0), \widehat{X}(0), \widehat{\zeta}^{2}(\cdot, 0), \widehat{\zeta}_{t}(\cdot, 0)\right)^{\top} \in \mathscr{H}^{2}$, system58admits a unique solution $\left(X(t), \zeta(\cdot, t), \zeta_{t}(\cdot, t)\right.$, $\left.\widehat{X}(t), \widehat{\zeta}(\cdot, t), \widehat{\zeta}_{t}(\cdot, t)\right)^{\top} \in C\left(0, \infty ; \mathscr{H}^{2}\right)$. Moreover, the solution of system58is exponentially stable in the sense that there exist two positive constants $M_{1}, \varepsilon_{1}$ such that

$$
\begin{aligned}
& \left\|\left(X(t), \zeta(\cdot, t), \zeta_{t}(\cdot, t), \widehat{X}(t), \widehat{\zeta}(\cdot, t), \widehat{\zeta}_{t}(\cdot, t)\right)^{\top}\right\|_{\mathscr{H}^{2}} \\
& \quad \leq M_{1} e^{-\varepsilon_{1} t}\left\|\left(X(0), \zeta(\cdot, 0), \zeta_{t}(\cdot, 0), \widehat{X}(0), \widehat{\zeta}(\cdot, 0), \widehat{\zeta}_{t}(\cdot, 0)\right)^{\top}\right\|_{\mathscr{H}^{2}}, \quad \forall t \geq 0 .
\end{aligned}
$$

Proof. By introducing error variable $\left(\widetilde{X}, \widetilde{\zeta}, \widetilde{\zeta}_{t}\right)$, we trans-

form system (58) into (44) and

$$
\left\{\begin{array}{l}
\dot{X}(t)=D X(t)+F \zeta(0, t), \\
\zeta_{t t}(x, t)=\zeta_{x x}(x, t)+q \zeta_{t}(0, t), \quad x \in(0,1) \\
\zeta_{x}(0, t)=0 \\
\zeta_{x}(1, t)=\int_{0}^{1}\left[k_{x}(1, y)+c_{1} l_{y y}(1, y)+c_{2} k(1, y)\right] \zeta(y, t) \mathrm{d} y+\int_{0}^{1}\left[l_{x}(1, y)+c_{1} k(1, y)+c_{2} l(1, y)\right] \zeta_{t}(y, t) \mathrm{d} y \\
+\left[\mu^{\prime}(1)+c_{1} l_{y}(1,0)+c_{1} \gamma(1) F+c_{2} \mu(1)\right] \zeta(0, t)+\left[c_{1} q \int_{0}^{1} l(1, y) \mathrm{d} y+c_{1} \mu(1)\right] \zeta_{t}(0, t) \\
-\left[c_{1} l_{y}(1,1)+c_{2}\right] \zeta(1, t)-c_{1} \zeta_{t}(1, t)+\left[c_{1} \gamma(1) D+c_{2} \gamma(1)+\gamma^{\prime}(1)\right] X(t)+f(t), \\
\zeta(x, 0)=\zeta_{0} \\
\zeta_{t}(x, 0)=\zeta_{1} \\
X(0)=X_{0} .
\end{array}\right.
$$


On account of transformation (3), system (60) can be mapped into the following target system:

$$
\left\{\begin{array}{l}
\dot{X}(t)=(D+F G) X(t)+F v(0, t), \\
v_{t t}(x, t)=v_{x x}(x, t), \\
v_{x}(0, t)=0, \\
v_{x}(1, t)=-c_{1} v_{t}(1, t)-c_{2} v(1, t)+f(t), \\
v(x, 0)=v_{0}(x), \\
v_{t}(x, 0)=v_{1}(x), \\
X(0)=X_{0} .
\end{array}\right.
$$

For " $v$ part", we can describe it into an evolutionary equation in $\mathscr{H}_{1}$ :

$$
\frac{\mathrm{d}}{\mathrm{d} t}\left(v(\cdot, t), v_{t}(\cdot, t)\right)^{\top}=\mathscr{A}_{1}\left(v(\cdot, t), v_{t}(\cdot, t)\right)^{\top}+\mathscr{B} f(t),
$$

where $\mathscr{B}=(0, \delta(x-1))^{\top}$ with $\delta(\cdot)$ being the Dirac distribution. Straightforward computation shows that

$$
\left\{\begin{array}{l}
\mathscr{A}_{1}^{*}(\phi, \psi)^{\top}=-\left(\psi, \phi^{\prime \prime}\right)^{\top}, \quad \forall(\phi, \psi)^{\top} \in D\left(\mathscr{A}_{1}^{*}\right), \\
D\left(\mathscr{A}_{1}^{*}\right)=\left\{(\phi, \psi)^{\top} \in H^{2}(0,1) \times H^{1}(0,1) \mid \mathscr{A}_{1}^{*}(\phi, \psi)^{\top} \in \mathscr{H}_{1}, \phi^{\prime}(0)=0, \phi^{\prime}(1)=c_{1} \psi(1)-c_{2} \phi(1)\right\} .
\end{array}\right.
$$

This is equivalent to saying that $\mathscr{B}^{*} \mathscr{A}_{1}^{*-1}$ is bounded and for any $T>0$, there exists $M_{T}>0$ depending on $T$, so that the system of the following:

$$
\left\{\begin{array}{l}
v_{t t}^{*}(x, t)=v_{x x}^{*}(x, t), \\
v_{x}^{*}(0, t)=0 \\
v_{x}^{*}(1, t)=-c_{1} v_{t}^{*}(1, t)-c_{2} v^{*}(1, t), \\
y_{0}^{*}=-v_{t}^{*}(1, t), \\
v^{*}(x, 0)=v_{0}^{*}(x), \\
v_{t}^{*}(x, 0)=v_{1}^{*}(x),
\end{array}\right.
$$

satisfies

$$
\int_{0}^{T}\left|v_{t}^{*}(1, t)\right|^{2} \leq M_{T} E^{*}(0)
$$

where

$$
E^{*}(t)=\frac{1}{2} \int_{0}^{1}\left(\left|v_{t}^{*}(x, t)\right|^{2}+\left|v_{x}^{*}(x, t)\right|^{2}\right) \mathrm{d} x+\frac{c_{2}}{2}\left|v^{*}(1, t)\right|^{2} .
$$

Actually,

$$
\begin{aligned}
\mathscr{A}_{1}^{*-1}(\phi, \psi)^{\top}= & \left(\int_{0}^{1} \int_{0}^{y} \psi(z) \mathrm{d} z \mathrm{~d} y+c_{2}^{-1} \int_{0}^{1} \psi(z) \mathrm{d} z\right. \\
& \left.-\frac{c_{1}}{c_{2}} \phi(1)-\int_{0}^{x} \int_{0}^{y} \psi(z) \mathrm{d} z \mathrm{~d} y,-\phi(x)\right)^{\top},
\end{aligned}
$$

$\mathscr{B}^{*} \mathscr{A}_{1}^{*-1}(\phi, \psi)^{\top}=-\phi(1)$.

Hence, $\mathscr{B}^{*} \mathscr{A}_{1}^{*-1}$ is bounded on $\mathscr{H}_{1}$. Taking derivative of (66), we have

$$
\dot{E}^{*}(t)=-c_{1}\left|v_{t}^{*}(1, t)\right|^{2} \leq 0
$$

Integrating above equation from 0 to $T$ with respect to $t$, we have

$$
\int_{0}^{T}\left|v_{t}^{*}(1, t)\right|^{2} \mathrm{~d} t \leq \frac{1}{c_{1}} E^{*}(0) .
$$

We have showed that $\mathscr{B}$ is admissible for $e^{\mathscr{A}_{1} t}$. Now the solution of (62) can be described as

$$
\begin{aligned}
\left(v(\cdot, t), v_{t}(\cdot, t)\right)^{\top}= & e^{\mathscr{A}_{1} t}\left(v_{0}, v_{1}\right)^{\top}+\int_{0}^{t} e^{\mathscr{A}_{1}(t-s)} \mathscr{B} r(s) \mathrm{d} s \\
& +\int_{0}^{t} e^{\mathscr{A}_{1}(t-s)} \mathscr{B} j(s) \mathrm{d} s,
\end{aligned}
$$

where $\int_{0}^{t} e^{\mathscr{A}_{1}(t-s)} \mathscr{B} j(s) \mathrm{d} s$ is exponentially stable and $\mathscr{B}$ is admissible for $e^{\mathscr{A}_{1} t}$. Since $e^{\mathscr{A}_{1} t}$ is exponentially stable, the first part on the right hand side in (70) decays exponentially. The exponential decay result of the second part can be obtained by the estimation of (62) in [36], noticing (48), (49), and $f(\cdot) \in L^{2}(0, \infty)$ in Theorem 2 .

Finally, the solution to system (60) can be obtained as

$$
\left(\zeta(\cdot, t), \zeta_{t}(\cdot, t)\right)^{\top}=P^{-1} P_{0}^{-1}\left(v(\cdot, t), v_{t}(\cdot, t)\right)^{\top},
$$

which decays exponential from the boundedness of operators $P^{-1}, P_{0}^{-1}$ and exponential stability of system (61). For " $X$ part," it admits a unique solution and the solution is exponentially stable by (38). (59) holds.

\section{Simulation Results}

In this section, we give some numerical examples for closedloop system (58) in order to prove the effectiveness of proposed feedback control law. We let these parameters be $q=2, c_{1}=1, c_{2}=1, c_{3}=1.2, c_{4}=0.5$ in system (58).

And the matrixes are given as follows: 


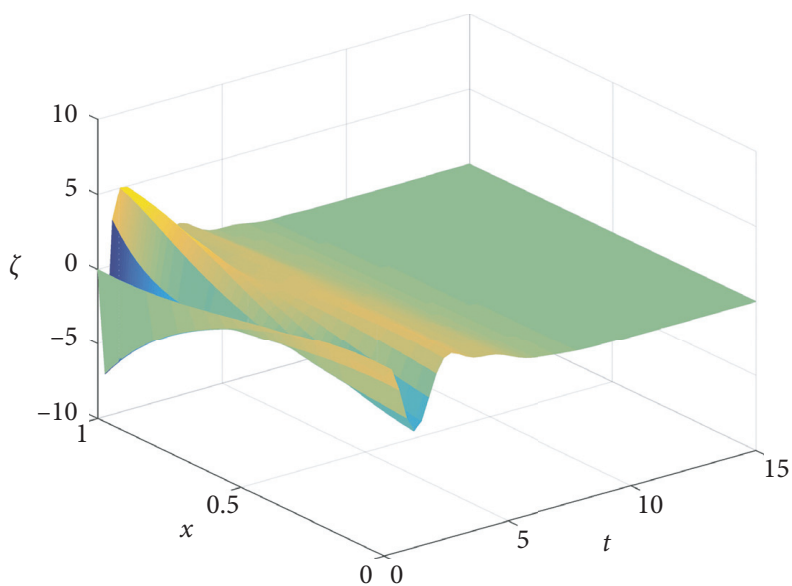

(a)

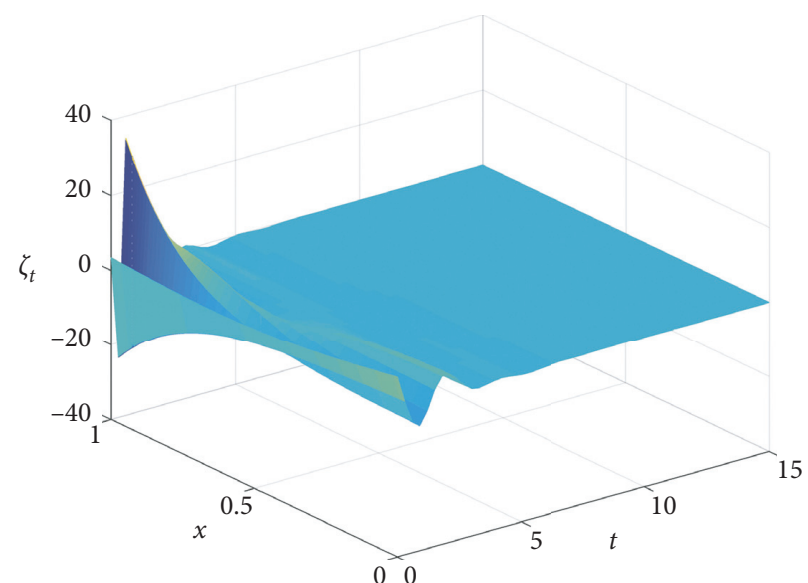

(b)

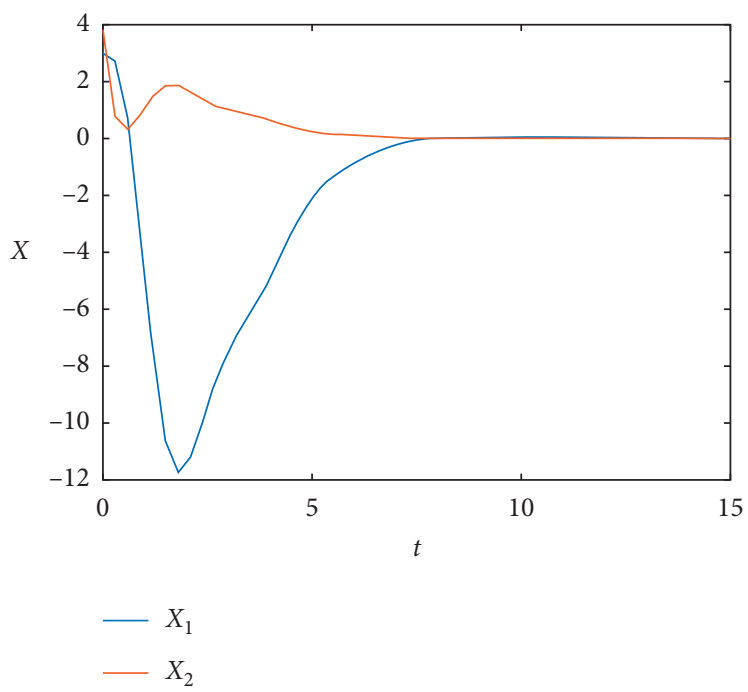

(c)

Figure 1: Solution of “ $\left(X, \zeta, \zeta_{t}\right)$ part” in the closed-loop system (58). (a) Displacement of the $\zeta$-part. (b) Velocity of the $\zeta$-part. (c) $X$-part.

$$
\begin{aligned}
& D=\left(\begin{array}{cc}
-1 & 1 \\
-1 & -10
\end{array}\right) \text {, } \\
& P=\left(\begin{array}{c}
4 \\
-2
\end{array}\right) \text {, } \\
& Q=\left(\begin{array}{ll}
-8 & -2
\end{array}\right) \text {, } \\
& F=\left(\begin{array}{c}
5 \\
-2
\end{array}\right) \text {, } \\
& G=\left(\begin{array}{ll}
-10 & -2
\end{array}\right) \text {. }
\end{aligned}
$$

We give the initial values:

$$
\begin{aligned}
\widehat{\zeta}_{0}(x) & =2 \cos 3 x, \\
\widehat{\zeta}_{1}(x) & =-2+3 \sin x, \\
\widehat{X} & =\left(\begin{array}{l}
1 \\
2
\end{array}\right), \\
\zeta_{0}(x) & =3+2 \sin 3 x, \\
\zeta_{1}(x) & =-2 x, \\
X & =\left(\begin{array}{l}
3 \\
4
\end{array}\right) .
\end{aligned}
$$

The solution about system (58) is shown in Figures 1 and 2. From Figure 1, we can see that the solution of " $\zeta$ part" in closed-loop system (58) decays rapidly. The image of " $X$ part" eventually tends to zero. In Figure 2, we 


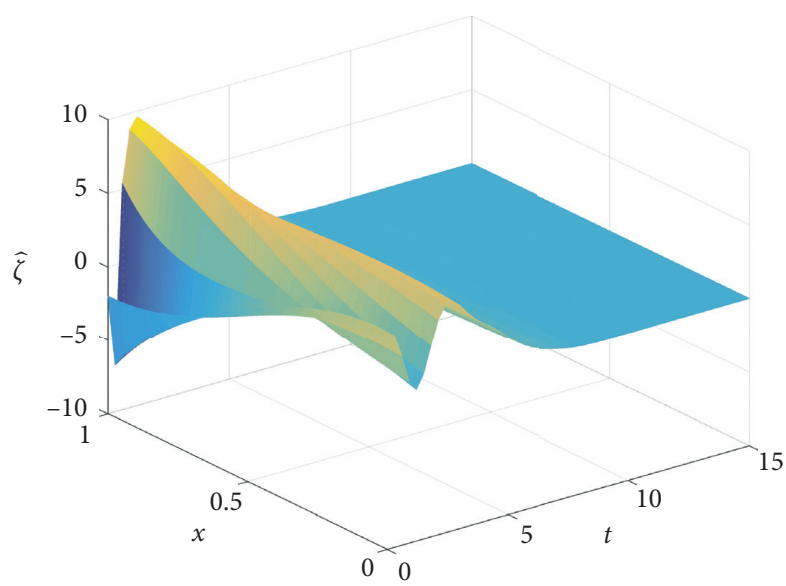

(a)

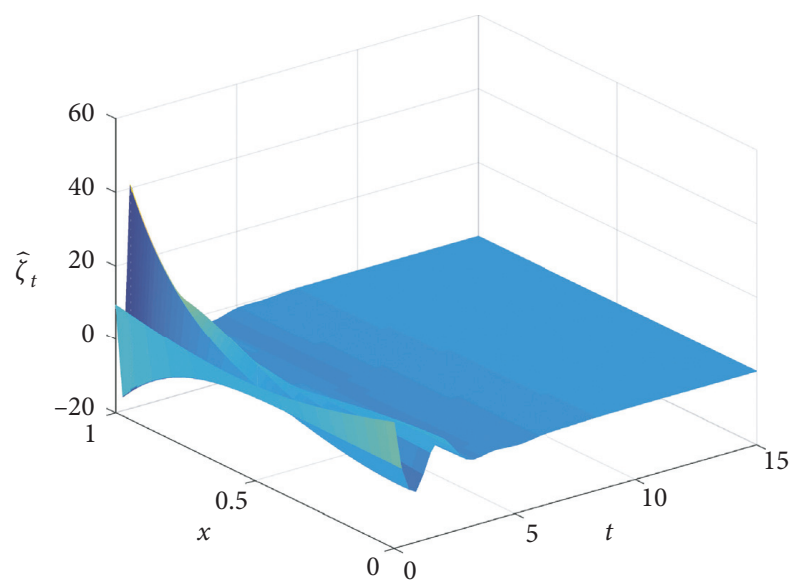

(b)

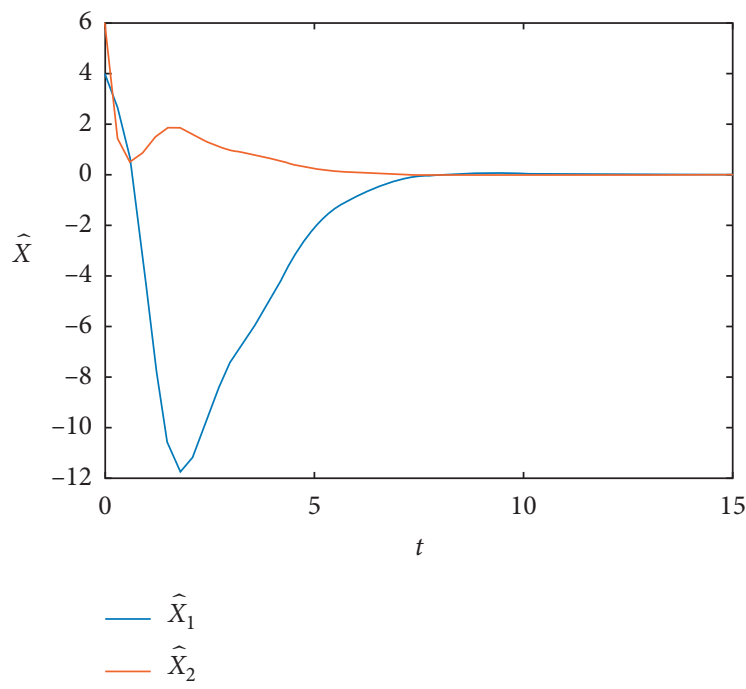

(c)

Figure 2: Solution of “ $\left(\widehat{X}, \widehat{\zeta}, \widehat{\zeta}_{t}\right)$ part” in the closed-loop system (58). (a) Displacement of the $\widehat{\varsigma}$-part. (b) Velocity of the $\widehat{\varsigma}$-part. (c) $\widehat{X}$-part.

notice that the result is similar to that in Figure 1. It explains the validity of our output feedback controller (57).

\section{Concluding Remarks}

This paper considers the boundary output feedback stabilization for a cascaded-wave PDE-ODE system with velocity recirculation. Inspired by Krstic and $\mathrm{Su}$ et al. $[30,33]$, we consider the problem of stabilization of a cascaded-wave PDE-ODE system with non-local term. We have used the backstepping method to turn system (1) into target system (3). Next we designed the state feedback controller and the output feedback controller for (1). And Theorems 1-3 have been proved. Finally, as shown in Figures 1 and 2, it is obvious that the output feedback control law (57) can stabilize system (1) exponentially by the measurements. Illustrative simulation results are given to demonstrate the validity of the theoretical analysis and show the performance of the proposed method. We hope to have more innovation and development about stabilization of PDE-ODE systems with more non-local terms in this field afterwards.

\section{Data Availability}

The data used to support the findings of this study are included within the article.

\section{Conflicts of Interest}

The authors declare that they have no conflicts of interest.

\section{Acknowledgments}

The authors are thankful for the support provided by the Key Project of National Natural Science Foundation of China (no. 62073203). 


\section{References}

[1] H. Feng, B.-Z. Guo, and X.-H. Wu, "Trajectory planning approach to output tracking for a 1-D wave equation," IEEE Transactions on Automatic Control, vol. 65, no. 5, pp. 1841$1854,2020$.

[2] B.-Z. Guo and F.-F. Jin, "Output feedback stabilization for one-dimensional wave equation subject to boundary disturbance," IEEE Transactions on Automatic Control, vol. 60, no. 3, pp. 824-830, 2015.

[3] B. Z. Guo and B. B. Xu, "A semi-discrete finite difference method to uniform stabilization of wave equation with local viscosity," IFAC Journal of Systems and Control, vol. 13, p. 101000, 2020.

[4] B.-Z. Guo and Z.-D. Mei, "Output feedback stabilization for a class of first-order equation setting of collocated well-posed linear systems with time delay in observation," IEEE Transactions on Automatic Control, vol. 65, no. 6, pp. 2612-2618, 2020.

[5] B. Z. Guo and H. J. Ren, "Stabilization and regularity transmission of a Schrodinger equation through boundary connections with a Kelvin-Voigt damped beam equation," Zeitschrift Fur Angewandte Mathematik und Mechanik, vol. 100, 2020.

[6] H. C. Zhou and B. Z. Guo, "Performance output tracking for one-dimensional wave equation subject to unmatched general disturbance and non-collocated control," European Journal of Control, vol. 39, pp. 39-52, 2018.

[7] H. C. Zhou and B. Z. Guo, "Unknown input observer design and output feedback stabilization for multi-dimensional wave equation with boundary control matched uncertainty," Journal of Differential Equations, vol. 263, pp. 2213-2246, 2017.

[8] X. H. Wu, H. Feng, and B. Z. Guo, "Output feedback stabilization for 1-D wave equation with variable coefficients and non-collocated observation," Systems and Control Letters, vol. 145, p. 104780, 2020.

[9] J. Li and Y. Liu, "Stabilization of coupled pde-ode systems with spatially varying coefficient," Journal of Systems Science and Complexity, vol. 26, no. 2, pp. 151-174, 2013.

[10] S. Tang and C. K. Xie, "Stabilization for a coupled PDE-ODE control system," Journal of the Franklin Institute, vol. 348, pp. 2142-2155, 2011.

[11] S. X. Tang and C. K. Xie, "State and output feedback boundary control for a coupled PDE-ODE system," Systems and Control Letters, vol. 60, pp. 540-545, 2011.

[12] S. X. Tang, C. K. Xie, and Z. C. Zhou, "Stabilization for a class of delayed coupled PDE-ODE systems with boundary control," in Proceedings of the Chinese Control And Decision Conference, Mianyang, China, November 2011.

[13] S. X. Tang and C. K. Xie, "Stabilization of a coupled PDE-ODE system by boundary control," in Proceedings of the 49th IEEE Conference on Decision and Control, pp. 4042-4047, Atlanta, GA, USA, December 2010.

[14] J. M. Wang, J. J. Liu, B. B. Ren, and J. H. Cheng, "Sliding mode control to stabilization of cascaded heat PDE-ODE systems subject to boundary control matched disturbance," Automatica, vol. 52, pp. 23-34, 2015.

[15] Y. L. Yuan, Z. W. Shen, and F. C. Liao, "Stabilization of coupled ODE-PDE system with intermediate point and spatially varying effects interconnection," Asian Journal of Control, vol. 19, 2016.
[16] Z. C. Zhou and C. Xu, "Stabilization of a second order ODEheat system coupling at intermediate point," Automatica, vol. 60, pp. 57-64, 2015.

[17] H. C. Zhou, B. Z. Guo, and Z. H. Wu, "Output feedback stabilisation for a cascaded wave PDE-ODE system subject to boundary control matched disturbance," International Journal of Control, vol. 89, pp. 2396-2405, 2016.

[18] Z. C. Zhou and S. X. Tang, "Boundary stabilization of a coupled wave-ODE system with internal anti-damping," International Journal of Control, vol. 85, pp. 1-11, 2012.

[19] F. Di Meglio, F. B. Argomedo, L. Hu, and M. Krstic, "Stabilization of coupled linear heterodirectional hyperbolic PDEODE systems," Automatica, vol. 87, pp. 281-289, 2018.

[20] W. He, T. T. Meng, X. Y. He, and S. Z. Sam Ge, "Unified iterative learning control for flexible structures with input constraints," Automatica, vol. 96, pp. 326-336, 2018.

[21] W. He and S. Z. Sam Ge, "Cooperative control of a nonuniform gantry crane with constrained tension," Automatica, vol. 66, pp. 146-154, 2016.

[22] S. Koga, D. Straub, M. Diagne, and M. Krstic, "Stabilization of filament production rate for screw extrusion-based polymer three-dimensional-printing," Journal of Dynamic Systems, Measurement, and Control, vol. 142, 2020.

[23] J. Wang and M. Krstic, "Delay-compensated control of sandwiched ODE-PDE-ODE hyperbolic systems for oil drilling and disaster relief," Automatica, vol. 120, 2020.

[24] J. Wang, S. Koga, Y. Pi, and M. Krstic, "Axial vibration suppression in a partial differential equation model of ascending mining cable elevator," Journal of Dynamic Systems, Measurement, and Control, vol. 140, 2018.

[25] S. X. Tang, L. Camacho-Solorio, Y. Wang, and M. Krstic, "State-of-charge estimation from a thermal Celectrochemical model of lithium-ion batteries," Automatica, vol. 83, pp. 206-219, 2017.

[26] H. Yu, M. Diagne, L. Zhang, and M. Krstic, "Bilateral boundary control of moving shockwave in LWR model of congested traffic," IEEE Transactions on Automatic Control, vol. 66, no. 3, pp. 1429-1436, 2021.

[27] M. Krstic, "Compensating actuator and sensor dynamics governed by diffusion PDEs," Systems \& Control Letters, vol. 58, no. 5, pp. 372-377, 2009.

[28] M. Krstic, "Compensating a string PDE in the actuation or sensing path of an unstable ODE," IEEE Transactions on Automatic Control, vol. 54, no. 6, pp. 1362-1368, 2009.

[29] M. Krstic and A. Smyshlyaev, "Backstepping boundary control for first-order hyperbolic PDEs and application to systems with actuator and sensor delays," Systems \& Control Letters, vol. 57, no. 9, pp. 750-758, 2008.

[30] M. Krstic, Delay Compensation for Nonlinear, Adaptive, and PDE Systems, pp. 270-277, Birkhauser, Basel, Switzerland, 2009.

[31] F.-F. Jin and W. Guo, "Boundary state feedback exponential stabilization for a one-dimensional wave equation with velocity recirculation," Automatica, vol. 113, p. 108796, 2020.

[32] A. Hasan and S. X. Tang, "Local exponential stabilization of a coupled burgers' PDE-ODE system," in Proceedings of the IEEE Conference on Decision and Control, Melbourne, Australia, December 2017.

[33] L. Su, W. Guo, J.-M. Wang, and M. Krstic, "Boundary stabilization of wave equation with velocity recirculation," IEEE Transactions on Automatic Control, vol. 62, no. 9, pp. 47604767, 2017.

[34] A. Smyshlyaev and M. Krstic, "Boundary control of an antistable wave equation with anti-damping on the uncontrolled 
boundary," Systems \& Control Letters, vol. 58, no. 8, pp. 617-623, 2009.

[35] B.-Z. Guo and C.-Z. Xu, "The stabilization of a one-dimensional wave equation by boundary feedback with noncollocated observation," IEEE Transactions on Automatic Control, vol. 52, no. 2, pp. 371-377, 2007.

[36] J. Liu and B.-Z. Guo, "A new semidiscretized order reduction finite difference scheme for uniform approximation of onedimensional wave equation," SIAM Journal on Control and Optimization, vol. 58, no. 4, pp. 2256-2287, 2020. 